\title{
Antimatter-Gravity Couplings, and Lorentz Symmetry
}

\author{
Jay D. Tasson
}

\begin{abstract}
Implications of possible CPT and Lorentz violation for antimattergravity experiments as well as other antimatter tests are considered in the context of the general field-theory-based framework of the Standard-Model Extension (SME).
\end{abstract}

Keywords Antimatter · Lorentz violation · Gravity

PACS 11.30.Cp $\cdot 04.80 . \mathrm{Cc} \cdot$ 11.30.Er

\section{Introduction}

The realm of antimatter is an area in which many predictions of our current best theories of physics, the Standard Model of particle physics and General Relativity remain unverified. Thus antimatter experiments provide the opportunity to place our existing theories on a stronger experimental foundation. Lorentz symmetry, along with the associated CPT symmetry [1], is an aspect of both of our existing theories that can be tested in antimatter experiments.

Beyond improving the experimental foundation of the Standard Model and General Relativity, antimatter experiments are among those that offer the potential to detect new physics at the Planck scale through searches for Lorentz and CPT violation with low-energy physics [2. Standard lore holds that our current theories are the low-energy limit of a more fundamental theory at the Planck scale. Candidates for the fundamental theory include suggestions such as string theory. The possibility of CPT and Lorentz violation has been shown

Proceedings of the 11th International Conference on Low Energy Antiproton Physics (LEAP2013) held in Uppsala, Sweden, 10-15 June 2013

Jay D. Tasson

Physics and Astronomy Department, Carleton College, Northfield, MN 55901, USA

Present Address:

Physics Department, St. Olaf College, Northfield, MN 55901, USA

E-mail: tasson1@stolaf.edu 
to arise in some candidates for the underlying theory including string theory [3] and other scenarios [4, hence searches for violations of these symmetries offers the opportunity to detect Planck-scale physics with existing technology [3].

The SME offers a comprehensive field-theoretic framework for investigating Lorentz and CPT symmetry as an expansion about known physics [5, 6, 7. As such, it contains known physics and offers the power to predict the outcome of relevant experiments. It should be emphasized that the SME is not a specific model, but a comprehensive test framework ideally suited for a broad search. Presently, no compelling evidence for CPT or Lorentz violation has been found. As such, a broad and systematic search may offer a more efficient way of seeking such violations than the consideration of many specific models having unclear relationships to each other. From this perspective, a few models that illustrate aspects of the general framework are useful; however, the time for more aggressive model building will come when new physics is found. This proceedings contribution reviews SME-based projects and proposals in the area of antimatter as well as an SME-based model that illustrates several possibilities in antimatter-gravity experiments.

\section{Basics}

The QED-extension limit of the gravitationally coupled SME [6] provides the basic theory relevant for the discussion to follow. The action for the theory can be schematically presented as

$$
S=S_{\psi}+S_{\text {gravity }}+S_{A} .
$$

Here the first term is the gravitationally coupled fermion sector, the puregravity sector is provided by the second term, and the final term is the photon sector. Each of the terms above consists of known physics along with all Lorentz-violating terms that can be constructed from the associated fields.

In this work we specialize to the popular minimal-SME limit, involving operators of dimension 3 and 4 . The minimal fermion-sector action can be presented explicitly as follows:

$$
S_{\psi}=\int d^{4} x\left(\frac{1}{2} i e e_{a}^{\mu} \bar{\psi} \Gamma^{a} \stackrel{\leftrightarrow}{D_{\mu}} \psi-e \bar{\psi} M \psi\right)
$$

where

$$
\begin{aligned}
\Gamma^{a} \equiv & \gamma^{a}-c_{\mu \nu} e^{\nu a} e^{\mu}{ }_{b} \gamma^{b}-d_{\mu \nu} e^{\nu a} e^{\mu}{ }_{b} \gamma_{5} \gamma^{b} \\
& -e_{\mu} e^{\mu a}-i f_{\mu} e^{\mu a} \gamma_{5}-\frac{1}{2} g_{\lambda \mu \nu} e^{\nu a} e^{\lambda}{ }_{b} e^{\mu}{ }_{c} \sigma^{b c}, \\
M \equiv & m+a_{\mu} e^{\mu}{ }_{a} \gamma^{a}+b_{\mu} e^{\mu}{ }_{a} \gamma_{5} \gamma^{a}+\frac{1}{2} H_{\mu \nu} e^{\mu}{ }_{a} e^{\nu}{ }_{b} \sigma^{a b},
\end{aligned}
$$

and $a_{\mu}, b_{\mu}, c_{\mu \nu}, d_{\mu \nu}, e_{\mu}, f_{\mu}, g_{\lambda \mu \nu}, H_{\mu \nu}$ are coefficient fields for Lorentz violation. Gravitational couplings occur here via the vierbein $e_{\mu}{ }^{a}$ and the covariant 
derivative. The Minkowski-spacetime fermion-sector action can be recovered in the limit $e_{\mu}{ }^{a} \rightarrow \delta_{\mu}^{a}$.

Leading contributions to the pure-gravity sector action take the form

$$
S_{\text {gravity }}=\frac{1}{2 \kappa} \int d^{4} x e\left(R-u R+s^{\mu \nu} R_{\mu \nu}^{T}+t^{\kappa \lambda \mu \nu} C_{\kappa \lambda \mu \nu}\right),
$$

where $R_{\mu \nu}^{T}$ is the traceless Ricci tensor, and $C_{\kappa \lambda \mu \nu}$ is the Weyl tensor. The first term provides the Einstein-Hilbert action. The coefficient field $s^{\mu \nu}$ in the third term is responsible for the relevant Lorentz-violating signals in the postnewtonian analysis 8]. The coefficient field $t^{\kappa \lambda \mu \nu}$ provides no contributions in the post-Newtonian analysis, while the coefficient field $u$ is not Lorentz violating. The photon sector, containing Maxwell electrodynamics along with associated Lorentz violation, is provided by the partial action $S_{A}$. Since it is not directly relevant for the discussion to follow, the explicit form of $S_{A}$ is omitted here, though in general it is of considerable interest and has been the subject of a large number of tests 9 .

The nature of the coefficient fields appearing above can be understood in two basic ways: explicit Lorentz violation and spontaneous Lorentz violation. In the context of explicit Lorentz violation, the content of the coefficient fields is imposed as an external choice, where as in the context of spontaneous Lorentz violation, the coefficient fields are dynamical fields within the theory that receive vacuum expectation values via the spontaneous breaking of Lorentz symmetry. Here spontaneous Lorentz violation can be thought of as analogous to the spontaneous breaking of $S U(2) \times U(1)$ symmetry in the Standard Model. Unlike electroweak-symmetry breaking, the vacuum values that arise from spontaneous Lorentz violation are vector or tensor objects known as coefficients for Lorentz violation, which can be thought of as establishing preferred directions in spacetime. For Minkowski-spacetime experiments seeking CPT- and Lorentz-violating effects associated with the vacuum values, the distinction between explicit and spontaneous Lorentz-symmetry breaking is not relevant, and spacetime-independent coefficients for Lorentz violation are typically assumed, both because they could be thought of as a leading term in an expansion of a more general function, and because energy and momentum are conserved in this limit.

The Minkowski-spacetime limit provides the relevant theory for the nongravitational tests considered in Sec. 3. In this limit, the pure-gravity sector is irrelevant, gravitational couplings in the fermion-sector 2 are neglected, and constant coefficients provide the relevant contribution to the coefficient fields. Theoretical tools for the analysis of a variety of experiments have been obtained from the general theory including the associated relativistic quantum mechanics [10, the corresponding nonrelativistic Hamiltonian [11], and the associated classical Lagrangian 12. Antimatter applications of these Minkowskispacetime results are considered in Sec. 3 .

In the context of gravitational studies, it has been shown that explicit Lorentz violation is typically incompatible with the Riemann geometry of existing gravity theories [6], though more general geometries may admit explicit 
breaking [13. Hence progress in gravitational studies requires consideration of spontaneous breaking. Here spacetime-independent vacuum values are still considered, but geometric consistency requires one to additionally consider the effects of certain contributions to the fluctuations about the vacuum values. Sec. 4 considers gravitational couplings. Lorentz-violating effects in a gravitational context can stem from the pure-gravity sector or gravitational couplings in the other sectors of the theory. Reference 8] provides numerous detailed experimental proposals for investigating the coefficient (vacuum value) $\bar{s}^{\mu \nu}$ associated with the coefficient field $s^{\mu \nu}$.

An analysis of gravitational couplings in the fermion sector is provided by Ref. [14. This includes the implications of the fluctuations in the coefficient fields that must be addressed in a gravitational analysis along with some additional theoretical issues. A detailed analysis of the implications of spin-independent coefficient fields $a_{\mu}, c_{\mu \nu}$, and $e_{\mu}$ is then provided for a large class of experiments. Vacuum values associated with these fields are denoted $\left(\bar{a}_{\text {eff }}\right)_{\mu}$, for the countershaded combination [15] associated with the $a_{\mu}$ and $e_{\mu}$ fields, and $\bar{c}_{\mu \nu}$ for the vacuum value associated with $c_{\mu \nu}$. The vacuum values introduced here correspond to the coefficients for Lorentz violation discussed in Minkowski spacetime. The experimental implications associated with these fermion-sector coefficients, including those relevant for antimatter, are summarized in Sec. 4.

\section{Nongravitational CPT Tests with Antimatter}

Over 1000 experimental and observational measurements have been performed in the context of the SME 9. Some methods associated with these measurements are based on CPT- and Lorentz-violating modifications to the energy levels of atoms [16, 17]. As the Earth revolves around the Sun and rotates on its axis, the orientation and boost of an experiment in the lab change. In the context of studies of Lorentz violation, such changes in boost and orientation imply annual and sidereal variations of energy levels. Since Lorentz-violating effects on various levels typically differ, Lorentz violation can be investigated by searching for relative changes in levels with time 16. A subset of the SME coefficients for Lorentz violation are also CPT violating, and the comparison of the spectrum of hydrogen with that of antihydrogen would also provide sensitivity to associated coefficients [17. An experiment aiming to obtain such sensitivity via a measurement of the hyperfine Zeeman line is presently in preparation $[18$.

Lorentz- and CPT-violating effects that are difficult to detect in matter experiments may in some cases be more readily seen in comparisons of matter and antimatter. The isotropic 'invisible' model (IIM) [14,19] was constructed as a special limit of the SME to illustrate a scenario in which Lorentz violation would produce a significantly larger effect in tests involving antimatter than in tests with only matter. The model is constructed by first noting that in any give inertial frame $O$, a subset of Lorentz-violating operators in the SME preserve 
rotational symmetry. Isotropic Lorentz violation is generated in that frame by setting the coefficients of the other operators to zero. In another frame $O^{\prime}$ that is boosted with respect to $O$, rotation invariance will be violated. The IIM then assumes that $\left(b^{p}\right)_{T^{\prime}}$ and isotropic $\left(d^{p}\right)_{\Xi^{\prime} \Xi^{\prime}}$ are the only nonzero coefficients for Lorentz violation in the CMB frame, and it further assumes $\left(b^{p}\right)_{T^{\prime}}=$ $k m^{p}\left(d^{p}\right)_{T^{\prime} T^{\prime}}$, for a suitable choice of constant $k$. Nonzero coefficients $\left(b^{p}\right)_{J}$ and $\left(d^{p}\right)_{J T}$ are then generated in the Sun-centered frame, which is boosted relative to the $\mathrm{CMB}$ frame. The dominant signals in terrestrial experiments with hydrogen are in the hyperfine structure and involve $\left(b^{p}\right)_{J}-m^{p}\left(d^{p}\right)_{J T}$, which vanishes for suitable $k$. Thus in the IIM, hydrogen experiments can only detect effects suppressed by at least one power of the boost of the Earth around the Sun, which is about $10^{-4}$ and requires experiments sensitive to annual variation. However, dominant effects in experiments with antihydrogen involve the combination $\left(b^{p}\right)_{J}+m^{p}\left(d^{p}\right)_{J T}$, since $b_{\mu}$ is a CPT odd coefficient, while $d_{\mu \nu}$ is CPT even. This implies unsuppressed signals would occur in the hyperfine structure of antihydrogen in the IIM, making it a field-theoretic toy model in which the effects of CPT and Lorentz violation would be at least $10^{4}$ times greater in antihydrogen than those in hydrogen or other nonrelativistic neutral matter.

The shifting of atomic energy levels discussed above constitute one method of searching for Lorentz and CPT violation in antimatter experiments. The comparison of energy levels for trapped particles with those of trapped antiparticles also leads to CPT-violation sensitivities in the SME [10. This method has led to experimental results for coefficients for Lorentz violation associated with the electron [20] and proton [21] and ongoing experiments provide the possibility of further results 22 .

\section{Gravitational Tests}

Sensitivity to coefficients $\left(\bar{a}_{\text {eff }}\right)_{\mu}, \bar{c}_{\mu \nu}$, and $\bar{s}_{\mu \nu}$ can be achieved via a wide variety of gravitational experiments [8,14] including gravimeter experiments [23], tests of the universality of free fall [24], redshift tests [25, spin-precession tests [26, experiments with devices traditionally used for short-range gravity tests [27, solar-system tests [28, gravity probe B [29], and binary pulsars [30]. The key point used in most laboratory tests is that the gravitational force acquires tiny corrections both along and perpendicular to the usual free-fall trajectory near the surface of the Earth due to the above coefficients. The effective inertial mass of a test body is also altered in a direction-dependent way by coefficients $\left(\bar{a}_{\text {eff }}\right)_{\mu}$ and $\bar{c}_{\mu \nu}$, resulting in a nontrivial relation between force and acceleration. These corrections are time dependent with variations at the annual and sidereal frequencies, and the corrections introduced by $\left(\bar{a}_{\text {eff }}\right)_{\mu}$ and $\bar{c}_{\mu \nu}$ are particle-species dependent.

The properties presented above lead naturally to a 4-category classification of laboratory tests that use Earth as a source. Monitoring the gravitational acceleration or force over time constitutes free-fall gravimeter tests or force- 
comparison gravimeter tests respectively. Similarly monitoring the relative acceleration of, or relative force on, a pair of test bodies constitutes a free-fall or a force-comparison Weak Equivalence Principle (WEP) test respectively. While such tests with ordinary, neutral matter yield numerous sensitivities to Lorentz violation, experimentally challenging versions of the tests highlighted above performed with antimatter, charged particles, and second- and thirdgeneration particles can yield sensitivities to Lorentz and CPT violation that are otherwise difficult or impossible to achieve. Gravitational experiments with antihydrogen $31,32,33,34,35,36,37$, charged-particle interferometry [38, 35, ballistic tests with charged particles [39], and signals in muonium free fall [40] are considered in Ref. 14. Here antimatter tests are considered in more detail.

An recent direct measurement of the fall of antihydrogen by the ALPHA collaboration has placed initial direct limits on differences in the freefall rate of matter and antimatter 31. A number of methods for improved measurements are in preparation or have been suggested including tests using a Moiré accelerometer 32, trapped antihydrogen 33, antihydrogen interferometry 34, 35], gravitational quantum states [36], and tests in space [37. As an example of the expected sensitivity of work in preparation, the AEGIS experiment 32 and the GBAR 33 . experiment both expect sensitivity at the percent level.

Antimatter-gravity experiments could obtain special sensitivities to the SME coefficients $\left(\bar{a}_{\mathrm{eff}}^{w}\right)_{\mu}$ and $\left(\bar{c}^{w}\right)_{\mu \nu}$. The key idea is that the sign of $\left(\bar{a}_{\mathrm{eff}}^{w}\right)_{\mu}$ reverses under CPT, while the sign of $\left(\bar{c}^{w}\right)_{\mu \nu}$ does not. Thus, antimatter experiments could in principle observe novel behaviors and could place cleaner constrains on certain combinations of SME coefficients than can be obtained with matter. The analysis of antimatter experiments is then the same as the treatment of the matter experiments discussed above with the only exception being the change in sign of $\left(\bar{a}_{\mathrm{eff}}^{w}\right)_{\mu}$ relative its matter counterpart.

In addition to providing a framework for the analysis of antimatter gravity experiments as highlighted above, the general field-theoretic approach of the SME illuminates some aspects of attempts to place indirect limits on the possibility of unconventional antimatter-gravity interactions that appear in the literature 41. Toy-model limits of the SME facilitate the discussion. One such model is the isotropic 'parachute' model (IPM) [14], which is similar in design to the IIM discussed above. The model is constructed by restricting the classical nonrelativistic Lagrange density of the SME in the Sun-centered frame $S$, to the limit in which only coefficients $\left(\bar{a}_{\text {eff }}^{w}\right)_{T}$ and isotropic $\left(\bar{c}^{w}\right)_{\Sigma \Xi}$ are nonzero. The effective classical Lagrangian for a test particle $\mathrm{T}$ moving in the gravitational field of a source $\mathrm{S}$ in this limit can be written in the suggestive form

$$
L_{\mathrm{IPM}}=\frac{1}{2} m_{i}^{\mathrm{T}} v^{2}+\frac{G_{N} m_{g}^{\mathrm{T}} m_{g}^{\mathrm{S}}}{r} .
$$

Here $m_{i}^{\mathrm{T}}$ is the effective inertial mass of $\mathrm{T}$, and $m_{g}^{\mathrm{T}}$ and $m_{g}^{\mathrm{S}}$ are the effective gravitational masses of $\mathrm{T}$ and $\mathrm{S}$, respectively. The effective masses are defined in terms of the coefficients for Lorentz violation $\left(\bar{a}_{\mathrm{eff}}^{w}\right)_{T},\left(\bar{c}^{w}\right)_{T T}$ and 
the conventional Lorentz invariant body masses $m^{\mathrm{B}}$ :

$$
\begin{aligned}
& m_{i}^{\mathrm{B}}=m^{\mathrm{B}}+\sum_{w} \frac{5}{3}\left(N^{w}+N^{\bar{w}}\right) m^{w}\left(\bar{c}^{w}\right)_{T T} \\
& m_{g}^{\mathrm{B}}=m^{\mathrm{B}}+\sum_{w}\left(\left(N^{w}+N^{\bar{w}}\right) m^{w}\left(\bar{c}^{w}\right)_{T T}+2 \alpha\left(N^{w}-N^{\bar{w}}\right)\left(\bar{a}_{\mathrm{eff}}^{w}\right)_{T}\right),
\end{aligned}
$$

where $\mathrm{B}$ is $\mathrm{T}$ or $\mathrm{S}, N^{w}$ and $N^{\bar{w}}$ denote the number of particles and antiparticles of type $w$, respectively, and $m^{w}$ is the mass of a particle of type $w$.

The IPM for electrons, protons, and neutrons, is then defined by the conditions

$$
\alpha\left(\bar{a}_{\mathrm{eff}}^{w}\right)_{T}=\frac{1}{3} m^{w}\left(\bar{c}^{w}\right)_{T T},
$$

where $w$ ranges over $e, p, n$. This condition ensure that for a matter body $\mathrm{B}$ the effective inertial and gravitational masses are equal. That is, $m_{i}^{\mathrm{B}}=$ $m_{g}^{\mathrm{B}}$, which implies that no Lorentz-violating effects appear in gravitational tests to third post-newtonian order using ordinary matter; however, for an antimatter test body T, $m_{i}^{\mathrm{T}} \neq m_{g}^{\mathrm{T}}$ within the IPM. Thus, observable signals may arise in comparisons of the gravitational responses of different types of antimatter or of the gravitational responses of matter and antimatter. The following paragraphs consider the implications for the IPM of some typical arguments against anomalous antimatter gravity as well as some new indirect limits recently published.

The question of whether energy remains conserved when matter and antimatter have different gravitational responses is one issue that has been raised 42. This point is moot in the context of the present SME-based discussion since the energy-momentum tensor is explicitly conserved. Still, consideration of the following thought experiment is instructive. Consider a particle and an antiparticle lowered in a gravitational field, converted to a photon pair, raised to the original location, and reconverted to the original particle-antiparticle pair. Arguments of this type normally proceed by assuming, for example, that the particle-antiparticle pair gain a particular amount of energy from the gravitational field as they fall, and this energy is converted to a pair of photons with no additional change in gravitational field energy. The photons are then assumed to couple differently to gravity than the particle-antiparticle pair, and hence they lose an amount of energy on the way back to the original height that differs from the amount gained by the particle-antiparticle pair on the way down, violating conservation of energy. To see explicitly how these issues are avoided in the IPM, first note that in the analysis of Ref. [14, the photons are conventional, partly via an available coordinate choice. Next, note that the CPT-odd coefficient $\left(\bar{a}_{\text {eff }}^{w}\right)_{T}$ shifts the effective gravitational coupling of the particles and the antiparticles by equal and opposite amounts relative to the photons. Thus $\left(\bar{a}_{\mathrm{eff}}^{w}\right)_{T}$ result in no net difference for the particle-antiparticle combination and the photons. To understand contributions due to the CPTeven coefficient $\left(\bar{c}^{w}\right)_{T T}$, note that the above argument assumes that no change in potential, or field energy occurs as the particle-antiparticle pair converts to a photon. If there is a change in gravitational couplings this will not typically 
be the case. Hence in the case of $\left(\bar{c}^{w}\right)_{T T}$ one finds that energy is conserved when the total energy of the system, including field energy, is considered.

Another indirect argument against anomalous antimatter gravity is based on neutral-meson systems, which provide natural interferometers mixing particle and antiparticle states 43 . Neutral-meson systems have already been used to place tight constraints on certain differences of the coefficients $\left(\bar{a}_{\mathrm{eff}}^{w}\right)_{\mu}$ for $w$ ranging over quark flavors via flat spacetime considerations [44,45]. No dominant implications arise from these constrains for baryons, which involve three valence quarks, or for leptons in the context of the IPM. Moreover, meson tests involve, valence $s, c$, or $b$ quarks, which are largely irrelevant for protons and neutrons. This same line of reasoning holds for gravitational interactions. Thus the flavor dependence of Lorentz and CPT violation in the SME implies that the IPM evades restrictions from meson systems.

As a final popular argument, consider the attempt to argue against anomalous antimatter gravity based on the large binding energy content of baryons, atoms, and bulk matter 46. A version of the argument relevant for the present discussion of antihydrogen could be constructed by noting that the quarks in hydrogen contain only about $10 \%$ of the mass, with much of the remainder contained in the gluon and sea binding. One might then concluding that the gravitational response of the two cannot differ by more than about $10 \%$ since the binding forces are comparable for hydrogen and antihydrogen. Such arguments implicitly assume that the gravitational response of a body is determined by its mass and hence by binding energy. In the context of the IPM, the coefficient $\left(\bar{a}_{\text {eff }}^{w}\right)_{T}$, leads to a correction to the gravitational force that is independent of mass, but can vary with flavor. Hence the Lorentz-violating modifications to the gravitational responses are determined primarily by the flavor content of the valence particles. One could even consider a scenario in which the anomalous gravitational effect is associated purely with the positron, as would occur in the IPM when $\left(\bar{a}_{\text {eff }}^{e}\right)_{T}$ is the only nonzero coefficient. A detailed consideration of radiative effects involving $\left(\bar{a}_{\text {eff }}^{w}\right)_{T},\left(\bar{c}^{w}\right)_{T T}$, and other SME coefficients for Lorentz violation [5,47] could result in more definite statements along the above lines, perhaps imposing the IPM condition only after renormalization; however, the essential points illustrated with the IPM are: the anomalous gravitational response of a body can be independent of mass, can vary with flavor, and can differ between particles and antiparticles.

Although the IPM provides an example of a field-theoretic toy model, which generates an anomalous gravitation response for antimatter that appears to evade many of the typical indirect limitations, the model can be limited by a rather different type of investigation with matter. Certain experiments with sensitivity to higher powers of velocity 14, including the recent redshift analysis [25], considerations of bound kinetic energy [24, and double-boost suppression terms, if analyzed, in some flat-spacetime tests can place constraints on the IPM. Presently the best constraints are based on bound kinetic energy and limit the anomalous gravitational response of antimatter in the IPM to parts in $10^{8} 24$. Note however, that these constraints are rather different from the usual arguments. It also may be possible to construct models similar to 
the IPM based on the recently analyzed higher-order terms in the SME [7], though this remains an open issue at present.

\section{Summary}

The search for Lorentz and CPT violation provides the opportunity to probe Planck-scale physics with existing technology, and the SME provides a general field-theoretic framework for such investigations. The comparison of matter and antimatter, provides a means of conducting such tests using spectroscopic and gravitational experiments. Moreover, a special limit of the SME provides a unique toy model, the IPM, for investigating indirect limits on antimatter gravity.

\section{References}

1. O.W. Greenberg, Phys. Rev. Lett. 89, 231602 (2002).

2. For a recent review, see, J.D. Tasson Rep. Prog. Phys. 77, 062901 (2014).

3. V.A. Kostelecký and S. Samuel, Phys. Rev. D 39, 683 (1989); V.A. Kostelecký and R. Potting, Nucl. Phys. B 359, 545 (1991).

4. N. Mavromatos, Hyperfine Interact. 228, 7 (2014), these proceedings.

5. D. Colladay and V.A. Kostelecký, Phys. Rev. D 55, 6760 (1997); Phys. Rev. D 58, 116002 (1998).

6. V.A. Kostelecký, Phys. Rev. D 69, 105009 (2004).

7. V.A. Kostelecký and M. Mewes, Phys. Rev. D 80, 015020 (2009); Phys. Rev. D 85, 096005 (2012); Phys. Rev. D 85, 096006 (2013).

8. Q.G. Bailey and V.A. Kostelecký, Phys. Rev. D 74, 045001 (2006).

9. Data Tables for Lorentz and CPT Violation, 2013 edition, V.A. Kostelecký and N. Russell, arXiv:0801.0287v6.

10. R. Bluhm, V.A. Kostelecký, and N. Russell, Phys. Rev. D 57, 3932 (1998).

11. V.A. Kostelecký and C.D. Lane, J. Math. Phys. 40, 6245 (1999).

12. V.A. Kostelecký and N. Russell, Phys. Lett. B 693, 443 (2010).

13. V.A. Kostelecký, Phys. Lett. B 701, 137 (2011).

14. V.A. Kostelecký and J.D. Tasson, Phys. Rev. D 83, 016013 (2011).

15. V.A. Kostelecký and J.D. Tasson, Phys. Rev. Lett. 102, 010402 (2009).

16. V.A. Kostelecký and C.D. Lane, Phys. Rev. D 60, 116010 (1999); R. Bluhm et al., Phys. Rev. Lett. 88, 090801 (2002).

17. R. Bluhm, V.A. Kostelecký, and N. Russell, Phys. Rev. Lett. 82, 2254 (1999).

18. C. Malbrunot, Hyperfine Interact. 228, 61 (2014), these proceedings; M. Hayden, these proceedings.

19. V.A. Kostelecký, unpublished (2003).

20. H. Dehmelt et al., Phys. Rev. Lett. 83, 4694 (1999).

21. G. Gabrielse et al., Phys. Rev. Lett. 82, 3198 (1999); these proceedings.

22. C.C. Rodegheri et al., Hyperfine Interact. 194, 93 (2009).

23. K.-Y. Chung et al., Phys. Rev. D 80, 016002 (2009)

24. M. Hohensee, H. Müller, and R.B. Wiringa Phys. Rev. Lett. 111, 151102 (2013).

25. H. Müller et al., Phys. Rev. Lett. 100, 031101 (2008); M. Hohensee et al., Phys. Rev. Lett. 106151102 (2011); Phys. Rev. Lett. 111, 050401 (2013).

26. J.D. Tasson Phys. Rev. D 86, 124021 (2012).

27. D. Bennett, V. Skavysh, and J. Long, in V.A. Kostelecký, ed., CPT and Lorentz Symmetry V, World Scientific, Singapore, 2010; H. Panjwani, L. Carbone, and C.C. Speake, in V.A. Kostelecký, ed., CPT and Lorentz Symmetry V, World Scientific, Singapore, 2010. 28. J.B.R. Battat, J.F. Chandler, and C.W. Stubbs, Phys. Rev. Lett. 99, 241103 (2007); A. Hees et al., arXiv:1301.1658 
29. Q.G. Bailey, R.D. Everett, and J.M. Overduin, Phys. Rev. D 88, 102001 (2013).

30. J.M. Weisberg in V.A. Kostelecký, ed., CPT and Lorentz Symmetry VI, World Scientific, Singapore, 2014.; L. Shao and N. Wex, Class. Quantum Grav. 30, 165020 (2013); L. Shao, Phys. Rev. Lett. 112, 111103 (2014).

31. C. Amole et al., Nat. Commun. 4, 1785 (2013); J. Fajans, these proceedings.

32. AEGIS Collaboration, A. Kellerbauer et al., Nucl. Instr. Meth. B 266, 351 (2008) A. Knecht et al., Hyperfine Interact. 228, 121 (2014), these proceedings; J. Storey et al., Hyperfine Interact. 228, 151 (2014), these proceedings.

33. P. Perez and Y. Sacquin, Class. Quantum Grav. 29, 184008 (2012); P. Indelicato et al., Hyperfine Interact. 228, 141 (2014), these proceedings.

34. AGE Collaboration, A.D. Cronin et al., Letter of Intent: Antimatter Gravity Experiment (AGE) at Fermilab, February 2009; D. Kaplan, arXiv:1007.4956.

35. P. Hamilton et al., arXiv:1308.1079.

36. A. Voronin et al., Hyperfine Interact. 228, 133 (2014), these proceedings.

37. F.M. Huber, E.W. Messerschmid, and G.A. Smith, Class. Quantum Grav. 18, 2457 (2001).

38. B. Neyenhuis, D. Christensen, and D.S. Durfee, Phys. Rev. Lett. 99, 200401 (2007).

39. F.S. Witteborn and W.M. Fairbank, Phys. Rev. Lett. 19, 1049 (1967).

40. K. Kirch, arXiv:physics/0702143 B. Lesche, Gen. Rel. Grav. 21, 623 (1989).

41. M.M. Nieto and T. Goldman, Phys. Rep. 205, 221 (1991).

42. P. Morrison, Am. J. Phys. 26, 358 (1958).

43. M.L. Good, Phys. Rev. 121, 311 (1961).

44. KTeV Collaboration, H. Nguyen, in V.A. Kostelecký, ed., CPT and Lorentz Symmetry II, World Scientific, Singapore, 2002 hep-ex/0112046]; A. Di Domenico, KLOE Collaboration, J. Phys. Conf. Ser. 171, 012008 (2009); FOCUS Collaboration, J.M. Link et al., Phys. Lett. B 556, 7 (2003); BaBar Collaboration, B. Aubert et al., Phys. Rev. Lett. 100, 131802 (2008); hep-ex/0607103

45. V.A. Kostelecký, Phys. Rev. Lett. 80, 1818 (1998); Phys. Rev. D 61, 016002 (2000); Phys. Rev. D 64, 076001 (2001); V.A. Kostelecký and R. Van Kooten, Phys. Rev. D 82, 101702 (2010).

46. L.I. Schiff, Phys. Rev. Lett. 1, 254 (1958), Proc. Natl. Acad. Sci. 45, 69 (1959).

47. R. Jackiw and V.A. Kostelecký, Phys. Rev. Lett. 82, 3572 (1999); M. Pérez-Victoria, JHEP 0104, 032 (2001); V.A. Kostelecký, C.D. Lane, and A.G.M. Pickering, Phys. Rev. D 65, 056006 (2002); V.A. Kostelecký and A.G.M. Pickering, Phys. Rev. Lett. 91, 031801 (2003); B. Altschul, Phys. Rev. D 69, 125009 (2004); Phys. Rev. D 70, 101701 (2004); B. Altschul and V.A. Kostelecký, Phys. Lett. B 628, 106 (2005); H. Belich et al., Eur. Phys. J. C 42, 127 (2005); T. Mariz et al., JHEP 0510, 019 (2005); G. de Berredo-Peixoto and I.L. Shapiro, Phys. Lett. B 642, 153 (2006); P. Arias et al., Phys. Rev. D 76, 025019 (2007); D. Colladay and P. McDonald, Phys. Rev. D 75, 105002 (2007); Phys. Rev. D 77, 085006 (2008); Phys. Rev. D 79, 125019 (2009); M. Gomes et al., Phys. Rev. D 78, 025029 (2009); D. Anselmi, Ann. Phys. 324, 874 (2009); Ann. Phys. 324, 1058 (2009). 